\title{
New Geraghty type contractions on metric-like spaces
}

\author{
Hassen Aydia, ${ }^{a, b}$ Abdelbasset Felhi ${ }^{\mathrm{c}, *}$, Hojjat Afshari ${ }^{\mathrm{d}}$ \\ a University of Dammam, Department of Mathematics, College of Education of Jubail, P. O. 12020, Industrial Jubail 31961 , Saudi Arabia. \\ ${ }^{b}$ Department of Medical Research, China Medical University Hospital, China Medical University, Taichung, Taiwan. \\ ${ }^{c}$ Department of Mathematics and Statistics, College of Sciences, King Faisal University, Hafouf, Saudi Arabia. \\ ${ }^{d}$ Faculty of Basic Science, University of Bonab, Bonab, Iran.
}

Communicated by W. Shatanawi

\begin{abstract}
Very recently, Fulga and Proca [A. Fulga, A. M. Proca, Abstr. Appl. Anal., (In press)] considered new Geraghty type contraction mappings and established a fixed point theorem for such mappings in complete metric spaces. In this paper, we prove the analogous result in the class of metric-like spaces which generalizes the main result of Karapinar et al. [E. Karapinar, H. H. Alsulami, M. Noorwali, Fixed Point Theory Appl., 2015 (2015), 22 pages]. We give some examples illustrating the presented result where [E. Karapınar, H. H. Alsulami, M. Noorwali, Fixed Point Theory Appl., 2015 (2015), 22 pages] is not applicable. An application is also provided. (C)2017 All rights reserved.
\end{abstract}

Keywords: Fixed point, Geraghty type contraction, metric-like space.

2010 MSC: 47H10, 54H25.

\section{Introduction and Preliminaries}

Fixed point theory is an essential tool to resolve many problems in different branches of mathematics, see $[7,11,22]$. Hitzler and Seda [12] are the first who considered the concept of metric-like (or dislocated metric) spaces. Later, Amini-Harandi [4] established some fixed point results in the class of metric-like spaces. Very recently, many fixed point results on metric-like spaces have been provided. For more details, see $[1-3,5,6,8,10,13,14,16-19,21,23-25]$.

Definition 1.1. Let $X$ be a nonempty set. A function $\sigma: X \times X \rightarrow[0, \infty)$ is said to be a metric-like (or a dislocated metric) on $X$ if for any $x, y, z \in X$, the following conditions hold:

$\left(\sigma_{1}\right) \sigma(x, y)=0 \Longrightarrow x=y$

$\left(\sigma_{2}\right) \sigma(x, y)=\sigma(y, x)$

\footnotetext{
*Corresponding author

Email addresses: hmaydi@uod.edu.sa (Hassen Aydi), afelhi@kfu.edu.sa (Abdelbasset Felhi), hojat.afshari@bonabu.ac.ir (Hojjat Afshari)
}

doi:10.22436/jnsa.010.02.38 
$\left(\sigma_{3}\right) \sigma(x, z) \leqslant \sigma(x, y)+\sigma(y, z)$.

The pair $(X, \sigma)$ is then called a metric-like (a dislocated metric) space.

Each metric-like $\sigma$ on $X$ generates a $T_{0}$ topology $\tau_{\sigma}$ on $X$ which has as a base the family open $\sigma$-balls $\left\{B_{\sigma}(x, \varepsilon): x \in X, \varepsilon>0\right\}$, where $B_{\sigma}(x, \varepsilon)=\{y \in X:|\sigma(x, y)-\sigma(x, x)|<\varepsilon\}$ for all $x \in X$ and $\varepsilon>0$.

Observe that a sequence $\left\{x_{n}\right\}$ in a metric-like space $(X, \sigma)$ converges to a point $x \in X$ with respect to $\tau_{\sigma}$, if and only if $\sigma(x, x)=\lim _{n \rightarrow \infty} \sigma\left(x, x_{n}\right)$.

Definition 1.2. Let $(X, \sigma)$ be a metric-like space.

(a) A sequence $\left\{x_{n}\right\}$ in $X$ is said to be a Cauchy sequence if $\lim _{n, m \rightarrow \infty} \sigma\left(x_{n}, x_{m}\right)$ exists and is finite.

(b) $(X, \sigma)$ is said to be complete if every Cauchy sequence $\left\{x_{n}\right\}$ in $X$ converges with respect to $\tau_{\sigma}$ to a point $x \in X$ such that $\lim _{n \rightarrow \infty} \sigma\left(x, x_{n}\right)=\sigma(x, x)=\lim _{n, m \rightarrow \infty} \sigma\left(x_{n}, x_{m}\right)$.

Lemma 1.3 ([17]). Let $(X, \sigma)$ be a metric-like space. Let $\left\{x_{n}\right\}$ be a sequence in $X$ such that $x_{n} \rightarrow x$, where $x \in X$ and $\sigma(x, x)=0$. Then, for all $y \in X$, we have

$$
\lim _{n \rightarrow \infty} \sigma\left(x_{n}, y\right)=\sigma(x, y) .
$$

Now let $\mathcal{F}$ be the family of all functions $\beta:[0, \infty) \rightarrow[0,1)$ which satisfy the condition

$$
\lim _{n \rightarrow \infty} \beta\left(t_{n}\right)=1 \text { implies } \lim _{n \rightarrow \infty} t_{n}=0 \text {. }
$$

In 2015, Karapinar et al. [15] proved the following particular result (it corresponds to $s=1$ and $\psi(t)=t$ in Corollary 3.7 in [15]).

Theorem $1.4([15])$. Let $(X, \sigma)$ be a complete metric-like space and $\mathrm{T}: \mathrm{X} \rightarrow \mathrm{X}$ be a mapping. Suppose that there exists $\beta \in \mathcal{F}$ such that

$$
\sigma(T x, T y) \leqslant \beta(\sigma(x, y)) \sigma(x, y),
$$

for all $x, y \in X$. Then $T$ has a unique fixed point $u \in X$ with $\sigma(u, u)=0$.

On the other hand, very recently Fulga and Proca [9] considered a new type of Geraghty contractions given as follows.

Definition 1.5. Let $(X, d)$ be a metric space. A mapping $T: X \rightarrow X$ is said to be a $\varphi_{E}$-Geraghty contraction on $(X, d)$ if there exists $\varphi \in \mathcal{F}$ such that:

$$
d(T x, T y) \leqslant \varphi(E(x, y)) E(x, y),
$$

for all $x, y \in X$, where $E(x, y)=d(x, y)+|d(x, T x)-d(y, T y)|$.

In this paper, we consider the analogous of Definition 1.5 in the class of metric-like spaces and we establish a fixed point result for this new Geraghty type contractive mapping. By presenting some illustrated examples, we will show that Theorem 1.4 is not applicable. At the end, we provide an application on the existence of a unique solution of an integral equation.

\section{Main results}

Our main result is as follows.

Theorem 2.1. Let $(X, \mathrm{~d})$ be a complete metric-like space and $\mathrm{T}: \mathrm{X} \rightarrow \mathrm{X}$ be a given mapping. Suppose there exists $\beta \in \mathcal{F}$ such that

$$
\sigma(T x, T y) \leqslant \beta(F(x, y)) F(x, y)
$$

for all $x, y \in X$, where $F(x, y)=\sigma(x, y)+|\sigma(x, T x)-\sigma(y, T y)|$. Then $T$ has a unique fixed point $u \in X$ with $\sigma(u, u)=0$. 
Proof. Let $x_{0} \in X$. We define a sequence $\left\{x_{n}\right\}$ in $X$ by $x_{n+1}=T x_{n}=T^{n+1} x_{0}$ for all $n \geqslant 0$. Suppose that $\sigma\left(x_{n_{0}}, x_{n_{0}+1}\right)=0$ for some $n_{0}$, so the proof is completed. Consequently, throughout the proof, we assume that

$$
\sigma\left(x_{n}, x_{n+1}\right) \neq 0 \text { for all } n \text {. }
$$

From (2.1), we have

$$
0<\sigma\left(x_{n}, x_{n+1}\right)=\sigma\left(T x_{n-1}, T x_{n}\right) \leqslant \beta\left(F\left(x_{n-1}, x_{n}\right)\right) F\left(x_{n-1}, x_{n}\right), \quad n \geqslant 1 .
$$

Note that

$$
F\left(x_{n-1}, x_{n}\right)=\sigma\left(x_{n-1}, x_{n}\right)+\left|\sigma\left(x_{n-1}, T x_{n-1}\right)-\sigma\left(x_{n}, T x_{n}\right)\right|=\sigma\left(x_{n-1}, x_{n}\right)+\left|\sigma\left(x_{n-1}, x_{n}\right)-\sigma\left(x_{n}, x_{n+1}\right)\right| .
$$

Take $\sigma_{n}=\sigma\left(x_{n-1}, x_{n}\right)$ and (2.2) becomes

$$
\sigma_{n+1} \leqslant \beta\left(\sigma_{n}+\left|\sigma_{n}-\sigma_{n+1}\right|\right)\left(\sigma_{n}+\left|\sigma_{n}-\sigma_{n+1}\right|\right) .
$$

Assume that there exists $n>0$ such that $\sigma_{n} \leqslant \sigma_{n+1}$. By (2.3), we get

$$
\sigma_{n+1} \leqslant \beta\left(\sigma_{n+1}\right) \sigma_{n+1}<\sigma_{n+1}
$$

which is a contradiction. Thus, for all $n>0, \sigma_{n}>\sigma_{n+1}$. Therefore (2.3) becomes

$$
0<\sigma_{n+1} \leqslant \beta\left(2 \sigma_{n}-\sigma_{n+1}\right)\left(2 \sigma_{n}-\sigma_{n+1}\right), \quad \forall n>0 .
$$

The real sequence $\left\{\sigma_{n}\right\}$ is decreasing, so there exists $\alpha$ such that $\lim _{n \rightarrow \infty} \sigma_{n}=\alpha$. Suppose that $\alpha>0$. Take $\mathrm{n} \rightarrow \infty$ in (2.4) to write

$$
\alpha=\lim _{n \rightarrow \infty} \sigma_{n+1} \leqslant \lim _{n \rightarrow \infty}\left[\beta\left(2 \sigma_{n}-\sigma_{n+1}\right)\left(2 \sigma_{n}-\sigma_{n+1}\right)\right] \leqslant \lim _{n \rightarrow \infty}\left(2 \sigma_{n}-\sigma_{n+1}\right)=\alpha .
$$

We obtain

$$
\lim _{n \rightarrow \infty} \beta\left(2 \sigma_{n}-\sigma_{n+1}\right)=1
$$

Since $\beta \in \mathcal{F}$, we get

$$
\alpha=\lim _{n \rightarrow \infty}\left(2 \sigma_{n}-\sigma_{n+1}\right)=0,
$$

which is a contradiction. Thus

$$
\lim _{n \rightarrow \infty} \sigma\left(x_{n}, x_{n+1}\right)=0
$$

We shall prove that $\left\{x_{n}\right\}$ is a Cauchy sequence in $(X, \sigma)$. We will prove that

$$
\lim _{n, m \rightarrow \infty} \sigma\left(x_{n}, x_{m}\right)=0
$$

We argue by contradiction. Then, there exists $\varepsilon>0$ for which we can find subsequences $\left\{x_{m(k)}\right\}$ and $\left\{x_{n(k)}\right\}$ of $\left\{x_{n}\right\}$ with $m(k)>n(k)>k$ such that for every $k$

$$
\sigma\left(x_{m(k)}, x_{n(k)}\right) \geqslant \varepsilon .
$$

Moreover, corresponding to $n(k)$ we can choose $m(k)$ in such a way that it is the smallest integer with $m(k)>n(k)$ and satisfying (2.7). Then

$$
\sigma\left(x_{m}(k)-1, x_{n(k)}\right)<\varepsilon .
$$

Using (2.7) and (2.8)

$$
\varepsilon \leqslant \sigma\left(x_{m(k)}, x_{n(k)}\right) \leqslant \sigma\left(x_{m(k)}, x_{m(k)-1}\right)+\sigma\left(x_{m(k)-1}, x_{n(k)}\right)<\sigma\left(x_{m(k)}, x_{m(k)-1}\right)+\varepsilon .
$$


By (2.5), we get

$$
\lim _{k \rightarrow \infty} \sigma\left(x_{m(k)}, x_{n(k)}\right)=\varepsilon .
$$

On the other hand, it is easy to see that

$$
\left|\sigma\left(x_{m(k)-1}, x_{n(k)-1}\right)-\sigma\left(x_{m(k)}, x_{n(k)}\right)\right| \leqslant \sigma\left(x_{m(k)-1}, x_{m(k)}\right)+\sigma\left(x_{n(k)}, x_{n(k)-1}\right) .
$$

Again, by (2.5) and (2.9),

$$
\lim _{k \rightarrow \infty} \sigma\left(x_{m(k)-1}, x_{n(k)-1}\right)=\varepsilon
$$

We go back to (2.1) to have

$$
\varepsilon \leqslant \sigma\left(x_{\mathfrak{m}(k)}, x_{\mathfrak{n}(k)}\right)=\sigma\left(T x_{\mathfrak{m}(k)-1}, T x_{n(k)-1}\right) \leqslant \beta\left(F\left(x_{\mathfrak{m}(k)-1}, x_{\mathfrak{n}(k)-1}\right)\right) . F\left(x_{m(k)-1}, x_{n(k)-1}\right),
$$

where

$$
F\left(x_{m(k)-1}, x_{n(k)-1}\right)=\sigma\left(x_{m(k)-1}, x_{n(k)-1}\right)+\left|\sigma\left(x_{m(k)-1}, x_{m(k)}\right)-\sigma\left(x_{n(k)-1}, x_{n(k)}\right)\right| .
$$

By (2.5) and (2.10),

$$
\lim _{k \rightarrow \infty} F\left(x_{m}(k)-1, x_{n}(k)-1\right)=\varepsilon
$$

We deduce

$$
\lim _{k \rightarrow \infty} \beta\left(F\left(x_{m(k)-1}, x_{n}(k)-1\right)\right)=1 .
$$

Since $\beta \in \mathcal{F}$, we have

$$
\lim _{k \rightarrow \infty} F\left(x_{m}(k)-1, x_{n}(k)-1\right)=0,
$$

which is a contradiction with respect to (2.11). Thus $\left\{x_{n}\right\}$ is Cauchy in the complete metric-like space $(X, \sigma)$. So there exists $u \in X$ such that

$$
\lim _{n \rightarrow \infty} \sigma\left(x_{n}, u\right)=\sigma(u, u)=\lim _{n, m \rightarrow \infty} \sigma\left(x_{n}, x_{m}\right)
$$

By (2.6), we write

$$
\lim _{n \rightarrow \infty} \sigma\left(x_{n}, u\right)=\sigma(u, u)=0 .
$$

We shall prove that $u$ is a fixed point of $T$. Assume that $u \neq T u$, so $\sigma(u, T u)>0$. From (2.1), we have

$$
\sigma\left(x_{n+1}, T u\right)=\sigma\left(T x_{n}, T u\right) \leqslant \beta\left(F\left(x_{n}, u\right)\right) . F\left(x_{n}, u\right) \leqslant F\left(x_{n}, u\right),
$$

where

$$
F\left(x_{n}, u\right)=\sigma\left(x_{n}, u\right)+\left|\sigma\left(x_{n}, x_{n+1}\right)-\sigma(u, T u)\right| \rightarrow \sigma(u, T u),
$$

as $n \rightarrow \infty$. Letting again $n \rightarrow \infty$ in (2.12) and using Lemma 1.3,

$$
0<\sigma(u, T u) \leqslant \lim _{n \rightarrow \infty} \beta\left(F\left(x_{n}, u\right)\right) \cdot \sigma(u, T u) \leqslant \sigma(u, T u) .
$$

So $\lim _{n \rightarrow \infty} \beta\left(F\left(x_{n}, u\right)\right)=1$ which implies that

$$
\lim _{n \rightarrow \infty} F\left(x_{n}, u\right)=0,
$$

which is a contradiction. Thus $\sigma(u, T u)=0$ and so $u=T u$, that is, $u$ is a fixed point of $T$ with $\sigma(u, u)=0$. We shall prove that such $u$ verifying $\sigma(u, u)=0$ is the unique fixed point of $T$. We argue by contradiction. Assume that there exists $u \neq v($ so $\sigma(u, v)>0)$ such that

$$
u=T u, \quad v=T v, \quad \sigma(u, u)=\sigma(v, v)=0 .
$$


We have

$$
F(u, v)=\sigma(u, v)+|\sigma(u, T u)-\sigma(v, T v)|=\sigma(u, v)+|\sigma(u, u)-\sigma(v, v)|=\sigma(u, v) .
$$

By (2.1), we get

$$
0<\sigma(u, v)=\sigma(T u, T v) \leqslant \beta(F(u, v)) . F(u, v)=\beta(\sigma(u, v)) . \sigma(u, v)<\sigma(u, v),
$$

which is a contradiction. Thus, there exists a unique $u \in X$ such that $u=T u$ with $\sigma(u, u)=0$.

Remark 2.2. Theorem 2.1 is the analogous of the main result of [9] in the class of metric-like spaces.

\section{Examples}

In this section, we present some examples where known results (in particular Theorem 1.4) in literature are not applicable.

Example 3.1. Let $X=\{0,1,2\}$ be endowed with the metric-like $\sigma$ given by

$$
\sigma(0,0)=\sigma(1,1)=0, \quad \sigma(2,2)=\frac{1}{2}, \quad \sigma(0,1)=\sigma(1,0)=1, \quad \sigma(0,2)=\sigma(2,0)=\frac{3}{2}, \quad \sigma(1,2)=\sigma(2,1)=\frac{8}{5} .
$$

Consider $\mathrm{T}: \mathrm{X} \rightarrow \mathrm{X}$ as $\mathrm{T} 0=\mathrm{T} 1=0$ and $\mathrm{T} 2=1$. Take

$$
\beta(t)= \begin{cases}\frac{1}{1+\frac{5}{11} t}, & \text { if } t>0, \\ \frac{1}{3}, & \text { if } t=0 .\end{cases}
$$

We shall prove that (2.1) holds. By symmetry of (2.1), we need the following cases:

Case 1: $(x, y)=(0,2)$. Since $F(0,2)=\frac{3}{2}+\left|0-\frac{8}{5}\right|=\frac{31}{10}$,

$$
\sigma(\mathrm{T} 0, \mathrm{~T} 2)=1 \leqslant \frac{341}{265}=\frac{1}{1+\frac{5}{11} \cdot \frac{31}{10}} \frac{31}{10}=\beta(\mathrm{F}(0,2)) . \mathrm{F}(0,2) .
$$

Case 2: $(x, y)=(1,2)$. We have $F(1,2)=\frac{8}{5}+\left|1-\frac{8}{5}\right|=\frac{11}{5}$. Also

$$
\sigma(\mathrm{T} 1, \mathrm{~T} 2)=1 \leqslant \frac{11}{10}=\frac{1}{1+\frac{5}{11} \cdot \frac{11}{5}} \frac{11}{5}=\beta(\mathrm{F}(0,2)) \cdot \mathrm{F}(0,2) .
$$

Case 3: $(x, y)=(0,1)$ or $x=y$. In this case, we have $\sigma(T x, T y)=0$.

So (2.1) holds for all $x, y \in X$. All hypotheses of Theorem 2.1 are satisfied, so $T$ has a unique fixed point, which is $u=0$ and $\sigma(0,0)=0$.

Note that Theorem 1.4 could not happen. Indeed, for $x=0$ and $y=2$, we have

$$
\sigma(\mathrm{T} 0, \mathrm{~T} 2)=1>\frac{33}{37}=\frac{1}{1+\frac{5}{11} \cdot \frac{3}{2}} \frac{3}{2}=\beta(\sigma(0,2)) \cdot \sigma(0,2) .
$$

Example 3.2. Take $X=[0,1]$. Consider the metric-like $\sigma: X \times X \rightarrow[0, \infty)$ as

$$
\sigma(x, y)=x+y \text {. }
$$

Let $\mathrm{T}: \mathrm{X} \rightarrow \mathrm{X}$ be defined by

$$
T x= \begin{cases}0, & \text { if } x \in\left[0, \frac{1}{2}\right), \\ \frac{1}{2 e^{2},} & \text { if } x \in\left[\frac{1}{2}, 1\right] .\end{cases}
$$

Take

$$
\beta(t)= \begin{cases}e^{-t}, & \text { if } t>0 \\ \frac{1}{2}, & \text { if } t=0\end{cases}
$$

We shall prove that (2.1) holds. We need the following cases: 
Case 1: $x, y \in\left[0, \frac{1}{2}\right)$. Here $T x=T y=0$, so (2.1) holds.

Case 2: $x \in\left[0, \frac{1}{2}\right)$ and $y \in\left[\frac{1}{2}, 1\right]$. We have

$$
F(x, y)=x+y+\left|x-\left(y+\frac{1}{2 e^{2}}\right)\right|=2 y+\frac{1}{2 e^{2}} .
$$

Then

$$
\sigma(T x, T y)=\frac{1}{2 e^{2}} \leqslant e^{-\left(2+\frac{1}{2 e^{2}}\right)} \cdot\left(1+\frac{1}{2 e^{2}}\right) \leqslant e^{-\left(2 y+\frac{1}{2 e^{2}}\right)} \cdot\left(2 y+\frac{1}{2 e^{2}}\right)=\beta(F(x, y)) \cdot F(x, y) .
$$

Case 3: $x, y \in\left[\frac{1}{2}, 1\right]$. Here we have

$$
F(x, y)=x+y+|x-y| .
$$

It suffices to treat the subcase $x \leqslant y$ (the other subcase is the same). So we have $F(x, y)=2 y$. Moreover

$$
\sigma(T x, T y)=\frac{1}{e^{2}}=e^{-2} \leqslant e^{-2 y} \cdot(2 y)=\beta(F(x, y)) . F(x, y) .
$$

In all cases, (2.1) holds for all $x, y \in X$. All hypotheses of Theorem 2.1 are satisfied, so $T$ has a unique fixed point, which is $u=0$ and $\sigma(0,0)=0$.

Example 3.3. Take $X=\{1,2,3\}$ and consider the metric-like $\sigma: X \times X \rightarrow[0, \infty)$ given by

$$
\sigma(1,1)=0, \quad \sigma(2,2)=1, \quad \sigma(3,3)=\frac{2}{3}, \quad \sigma(1,2)=\sigma(2,1)=\frac{3}{5}, \quad \sigma(2,3)=\sigma(3,2)=\frac{4}{5}, \quad \sigma(1,3)=\sigma(3,1)=\frac{7}{10} .
$$

Since $\sigma(1,1) \neq 0$, so $\sigma$ is not a metric. Consider $\mathrm{T} 1=\mathrm{T} 3=1$ and $\mathrm{T} 2=3$. Take $\beta(\mathrm{t})=\frac{8}{9}$. We distinguish the following cases:

Case 1: $(x, y)=(1,2)$. We have $F(x, y)=\frac{7}{5}$. Then

$$
\sigma(T x, T y)=\frac{7}{10} \leqslant \frac{8}{9} \cdot \frac{7}{5}=\beta(F(x, y)) \cdot F(x, y)
$$

Case 2: $(x, y)=(2,3)$. We have $F(x, y)=\frac{9}{10}$. Then

$$
\sigma(T x, T y)=\frac{7}{10} \leqslant \frac{8}{9} \cdot \frac{9}{10}=\beta(F(x, y)) \cdot F(x, y)
$$

Case 3: $(x, y)=(1,3)$ or $x=y$. We have $\sigma(T x, T y)=0$.

By symmetry, (2.1) holds for all $x, y \in X$. All hypotheses of Theorem 2.1 hold, so $T$ has a unique fixed point, which is $u=1$ and $\sigma(1,1)=0$.

Mention that Theorem 1.4 is not applicable for any $\beta \in \mathcal{F}$. Indeed, for $x=1$ and $y=2$, we have

$$
\sigma(\mathrm{T} 1, \mathrm{~T} 2)=\frac{7}{10}>\frac{3}{5} \geqslant \beta(\sigma(1,2)) \cdot \sigma(1,2) .
$$

\section{Application}

Let $X=C([0,1], \mathbb{R})$ be the set of real continuous functions defined on $[0,1]$. Take the metric-like $\sigma: X \times X \rightarrow[0, \infty)$ given by

$$
\sigma(x, y)=\|(x-y)\|_{\infty}=\sup _{t \in[0,1]}|x(t)-y(t)|,
$$

for all $x, y \in X$. Then $(X, \sigma)$ is a complete metric-like space. We consider the following integral equation

$$
x(t)=P(t)+\int_{0}^{1} S(t, u) f(u, x(u)) d u, \quad t \in[0,1],
$$


where $f:[0,1] \times \mathbb{R} \rightarrow \mathbb{R}$ and $P:[0,1] \rightarrow \mathbb{R}$ are two continuous functions and $S:[0,1] \times[0,1] \rightarrow[0, \infty)$ is a function such that $S(t,.) \in \mathrm{L}^{1}([0,1])$ for all $t \in[0,1]$.

Consider the operator $\mathrm{T}: \mathrm{X} \rightarrow \mathrm{X}$ defined by

$$
T(x)(t)=P(t)+\int_{0}^{1} S(t, u) f(u, x(u)) d u, \quad t \in[0,1] .
$$

Theorem 4.1. Suppose that the following conditions are satisfied:

(i) there exists $\eta: X \times X \rightarrow[0, \infty)$ such that for all $u \in[0,1]$ and for all $x, y \in X$

$$
0 \leqslant|f(u, x(u))-f(u, y(u))| \leqslant \eta(x, y)|x(u)-y(u)|,
$$

(ii) there exists $\beta:[0, \infty) \rightarrow[0,1)$ such that

$$
\lim _{n \rightarrow \infty} \beta\left(t_{n}\right)=1 \text { implies } \lim _{n \rightarrow \infty} t_{n}=0,
$$

and

$$
\left\|\int_{0}^{1} S(t, u) \eta(x, y) d u\right\|_{\infty} \leqslant \beta\left(\|x-y\|_{\infty}+\|x-T x\|_{\infty}+\|y-T y\|_{\infty}\right) .
$$

Then the integral equation (4.1) has a unique solution in X.

Proof. Clearly, any fixed point of (4.2) is a solution of (4.1). By conditions (i) and (ii), we obtain

$$
\begin{aligned}
|T(x)(t)-T(y)(t)| & =\left|\int_{0}^{1} S(t, u)[f(u, x(u))-f(u, y(u))] d u\right| \\
& \leqslant \int_{0}^{1} S(t, u)|f(u, x(u))-f(u, y(u))| d u \\
& \leqslant \int_{0}^{1} S(t, u) \eta(x, y)|x(u)-y(u)| d u \\
& \leqslant \int_{0}^{1} S(t, u) \eta(x, y)\|x-y\|_{\infty} d u \\
& \leqslant F(x, y) \int_{0}^{1} S(t, u) \eta(x, y) d u \\
& \leqslant F(x, y) \beta(F(x, y))
\end{aligned}
$$

where $F(x, y)=\|x-y\|_{\infty}+\|x-T x\|_{\infty}+\|y-T y\|_{\infty}$. Then we have

$$
\|(T(x)-T(y))\|_{\infty} \leqslant F(x, y) \beta(F(x, y)) .
$$

Thus for all $x, y \in X$, we obtain

$$
\sigma((T(x), T(y))) \leqslant F(x, y) \beta(F(x, y)) .
$$

This implies that the hypotheses of Theorem 2.1 hold. Thus the operator T has a unique fixed point, that is, the integral equation (4.2) has a unique solution in $X$.

We provide the following example illustrating Theorem 4.1.

Example 4.2. Consider the following functional integral equation

$$
x(t)=\frac{t^{2}}{1+t^{2}}+\frac{1}{27} \int_{0}^{1} \frac{u \cos t}{54(1+t)} \frac{|x(u)|}{1+|x(u)|} d u,
$$


for $t \in[0,1]$. Observe that this equation is a special case of (4.1) with

$$
P(t)=\frac{t^{2}}{1+t^{2}}, \quad S(t, u)=\frac{u}{3(1+t)}, \quad f(t, x)=\frac{\cos t}{18} \frac{|x|}{(1+|x|)} .
$$

Consider the operator $\mathrm{T}: \mathrm{X} \rightarrow \mathrm{X}$ defined by

$$
T(x)(t)=P(t)+\int_{0}^{1} S(t, u) f(u, x(u)) d u, t \in[0,1] .
$$

Moreover, take $\beta:[0, \infty) \rightarrow[0,1)$ by the following

$$
\beta(t)=\frac{1}{2} \text { for } t=0 \text { and } \beta(t)=\frac{t^{2}+1}{2 t^{2}+1} \text { for } t>0 .
$$

Let $\eta(x, y)=1$. For arbitrary fixed $x, y \in \mathbb{R}$, we obtain

$$
|f(t, x)-f(t, y)|=\left|\frac{\cos t}{18} \frac{|x|}{(1+|x|)}-\frac{\cos t}{18} \frac{|y|}{(1+|y|)}\right| \leqslant \frac{1}{18}|x-y| \leqslant|x-y|
$$

and

$$
\left\|\int_{0}^{1} S(t, u) \eta(x, y) d u\right\|_{\infty}=\frac{1}{6} \leqslant \beta\left(\|x-y\|_{\infty}+\|x-T x\|_{\infty}+\|y-T y\|_{\infty}\right) .
$$

By using Theorem 4.1, the integral equation (4.3) has a unique solution in $C([0,1], \mathbb{R})$.

\section{References}

[1] C. T. Aage, J. N. Salunke, The results on fixed points in dislocated and dislocated quasi-metric space, Appl. Math. Sci. (Ruse), 59 (2008), 2941-2948. 1

[2] S. A. Al-Mezel, C.-M. Chen, E. Karapınar, V. Rakočević, Fixed point results for various $\alpha$-admissible contractive mappings on metric-like spaces, Abstr. Appl. Anal., 2014 (2014), 15 pages.

[3] H. H. Alsulami, E. Karapınar, H. Piri, Fixed points of modified F-contractive mappings in complete metric-like spaces, J. Funct. Spaces, 2015 (2015), 9 pages. 1

[4] A. Amini-Harandi, Metric-like spaces, partial metric spaces and fixed points, Fixed Point Theory Appl., 2012 (2012), 10 pages. 1

[5] H. Aydi, A. Felhi, S. Sahmim, Fixed points of multivalued nonself almost contractions in metric-like spaces, Math. Sci. (Springer), 9 (2015), 103-108. 1

[6] H. Aydi, E. Karapınar, Fixed point results for generalized $\alpha-\psi$-contractions in metric-like spaces and applications, Electron. J. Differential Equations, 2015 (2015), 15 pages. 1

[7] H. Aydi, E. Karapınar, S. Rezapour, A generalized Meir-Keeler-type contraction on partial metric spaces, Abstr. Appl. Anal., 2012 (2012), 10 pages. 1

[8] R. D. Daheriya, R. Jain, M. Ughade, Some fixed point theorem for expansive type mapping in dislocated metric space, ISRN Math. Anal., 2012 (2012), 5 pages. 1

[9] A. Fulga, A. M. Proca, Fixed points for $\varphi_{\mathrm{E}}$-Geraghty contractions, Abstr. Appl. Anal., (In press). 1, 2.2

[10] R. George, R. Rajagopalan, S. Vinayagam, Cyclic contractions and fixed points in dislocated metric spaces, Int. J. Math. Anal. (Ruse), 9 (2013), 403-411. 1

[11] V. Gupta, W. Shatanawi, N. Mani, Fixed point theorems for $(\Psi, \beta)$-Geraghty contraction type maps in ordered metric spaces and some applications to integral and ordinary differential equations, J. Fixed Point Theory Appl., 2016 (2016), 17 pages. 1

[12] P. Hitzler, A. K. Seda, Dislocated topologies, J. Electr. Eng., 51 (2000), 3-7. 1

[13] N. Hussain, J. R. Roshan, V. Parvaneh, Z. Kadelburg, Fixed points of contractive mappings in b-metric-like spaces, Scientific World J., 2014 (2014), 15 pages. 1

[14] A. Isufati, Fixed point theorems in dislocated quasi-metric space, Appl. Math. Sci. (Ruse), 5 (2010), 217-233. 1

[15] E. Karapinar, H. H. Alsulami, M. Noorwali, Some extensions for Geragthy type contractive mappings, Fixed Point Theory Appl., 2015 (2015), 22 pages. 1, 1.4

[16] E. Karapınar, H. Aydi, A. Felhi, S. Sahmim, Hausdorff metric-like, generalized Nadler's Fixed Point Theorem on MetricLike Spaces and application, Miskolc Math. Notes, (In press). 1 
[17] E. Karapınar, P. Salimi, Dislocated metric space to metric spaces with some fixed point theorems, Fixed Point Theory Appl., 2013 (2013), 19 pages. 1.3

[18] P. S. Kumari, Some fixed point theorems in generalized dislocated metric spaces, Math. Theory Model., 1 (2011), 16-22.

[19] P. S. Kumari, V. V. Kumar, I. Rambhadra Sarma, Common fixed point theorems on weakly compatible maps on dislocated metric spaces, Math. Sci. (Springer), 6 (2012), 5 pages. 1

[20] S. G. Matthews, Partial metric topology, Papers on general topology and applications, Flushing, NY, (1992), Ann. New York Acad. Sci., New York Acad. Sci., New York, 728 (1994), 183-197.

[21] I. R. Sarma, P. S. Kumari, On dislocated metric spaces, Int. J. Math. Arch., 1 (2012), 72-77. 1

[22] W. Shatanawi, M. S. MD. Noorani, H. Alsamir, A. Bataihah, Fixed and common fixed point theorems in partially ordered quasi-metric spaces, J. Math. Computer Sci., 16 (2016), 516-528. 1

[23] R. Shrivastava, Z. K. Ansari, M. Sharma, Some results on fixed points in dislocated and dislocated quasi-metric spaces, J. Adv. Stud. Topol., 3 (2012), 25-31. 1

[24] M. Shrivastava, K. Qureshi, A. D. Singh, A fixed point theorem for continuous mapping in dislocated quasi-metric spaces, Int. J. Theor. Appl. Sci., 4 (2012), 39-40.

[25] K. Zoto, E. Hoxha, A. Isufati, Some new results in dislocated and dislocated quasi-metric spaces, Appl. Math. Sci. (Ruse), 6 (2012), 3519-3526. 1 